\title{
Iraq: Statistical Appendix
}

This Statistical Appendix paper for Iraq was prepared by a staff team of the International Monetary Fund as background documentation for the periodic consultation with the member country. It is based on the information available at the time it was completed on July 18, 2007. The views expressed in this document are those of the staff team and do not necessarily reflect the views of the government of Iraq or the Executive Board of the IMF.

The policy of publication of staff reports and other documents by the IMF allows for the deletion of market-sensitive information.

To assist the IMF in evaluating the publication policy, reader comments are invited and may be sent by e-mail to publicationpolicy@imf.org.

Copies of this report are available to the public from

International Monetary Fund • Publication Services

$70019^{\text {th }}$ Street, N.W. • Washington, D.C. 20431

Telephone: (202) 623-7430 • Telefax: (202) 623-7201

E-mail: publications@imf.org •Internet: http://www.imf.org

Price: $\$ 18.00$ a copy

\section{International Monetary Fund Washington, D.C.}





\section{INTERNATIONAL MONETARY FUND}

\section{IRAQ}

\section{Statistical Appendix}

\section{Approved by Middle East and Central Asia Department}

July 18,2007

Contents

Statistical Appendix Tables

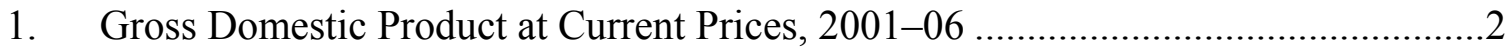

2. Gross Domestic Product at Constant 1998 Prices, 2000-06 ........................................

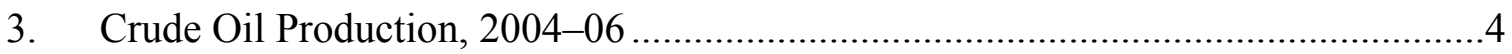

4. Electricity-Average Monthly Production, 2001-06 ..................................................5

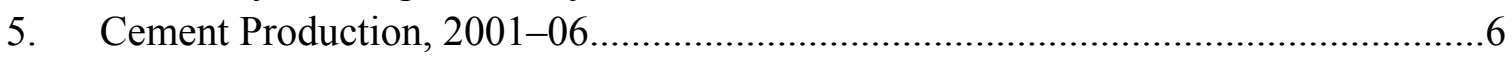

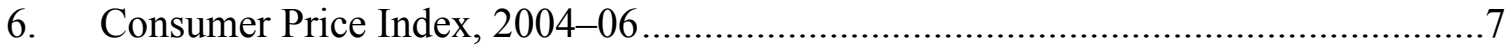

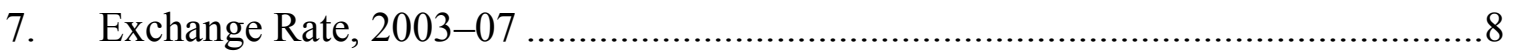

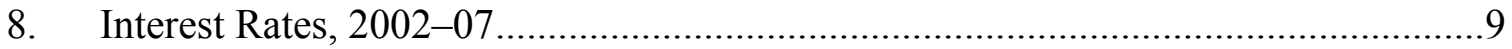

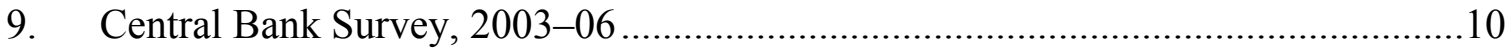

10. Depository Corporations Survey, 2004-06 ………...........................................

11. Fiscal and Oil Sector Accounts, 2004-06............................................................12

12. Government Current Expenditure by Source, 2004-06 ........................................13

13. Government Capital Expenditure by Source, 2004-06 ……………………...........14

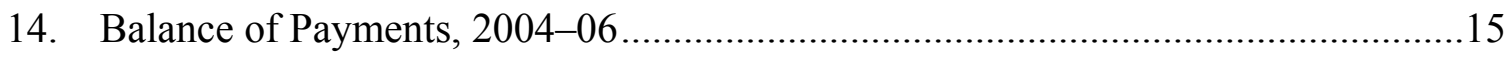

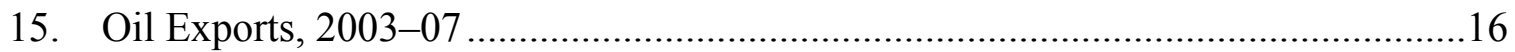

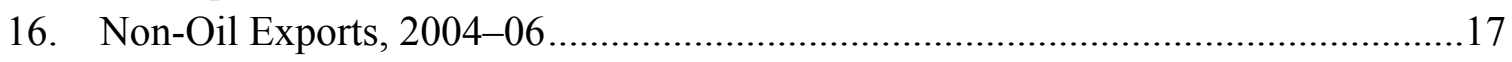

17. Exports by Countries of Destination and SITC Classification, 2003-06 ….............18

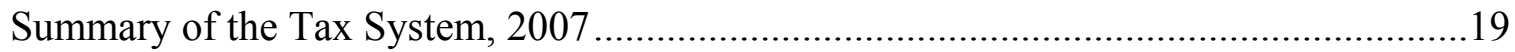


Table 1. Iraq: Gross Domestic Product at Current Prices, 2001-06 (In billions of ID, unless otherwise indicated)

\begin{tabular}{|c|c|c|c|c|c|c|}
\hline & 2001 & $2002^{*}$ & $2003^{*}$ & $2004^{*}$ & $2005^{*}$ & $2006^{*}$ \\
\hline Agriculture, forestry, hunting, and fishing & 2,863 & 3,513 & 2,487 & 3,539 & 4,249 & 4,133 \\
\hline Mining and quarrying & 30,817 & 29,045 & 20,372 & 30,543 & 39,366 & 50,302 \\
\hline Crude oil & 30,799 & 29,021 & 20,350 & 30,496 & 39,316 & 50,240 \\
\hline Other types of mining & 18 & 23 & 23 & 47 & 50 & 62 \\
\hline Manufacturing industry & 610 & 624 & 304 & 771 & 1,221 & 1,288 \\
\hline Electricity and water & 74 & 79 & 65 & 263 & 393 & 367 \\
\hline Building and construction & 485 & 674 & 217 & 468 & 2,932 & 616 \\
\hline Transport, communications, and storage & 2,610 & 3,238 & 2,284 & 3,688 & 4,911 & 5,562 \\
\hline Wholesale, retail, hotels \& others & 2,621 & 2,546 & 1,915 & 3,071 & 4,084 & 4,630 \\
\hline Finance, insurance, real estate, and business services & 410 & 478 & 391 & 663 & 931 & 840 \\
\hline Banking and insurance & 207 & 255 & 161 & 322 & 521 & 372 \\
\hline Ownership of dwelling & 204 & 222 & 230 & 341 & 410 & 468 \\
\hline Social and personal services & 1,004 & 1,047 & 1,859 & 5,200 & 6,140 & 9,983 \\
\hline Social services & 595 & 594 & 1,332 & 4,542 & 5,253 & 9,002 \\
\hline Personal services & 409 & 453 & 527 & 658 & 887 & 981 \\
\hline Total by activities & 41,494 & 41,243 & 29,894 & 48,207 & 64,228 & 77,724 \\
\hline Less: Imputed banks service charge & 180 & 220 & 309 & 248 & 227 & 357 \\
\hline GDP at current factor cost & 41,315 & 41,023 & 29,586 & 47,959 & 64,000 & 77,367 \\
\hline Plus: Indirect taxes & 546 & 405 & 187 & 246 & 777 & 1,260 \\
\hline Less: Subsidies & 7,752 & 7,304 & 9,210 & 11,155 & 14,786 & 16,781 \\
\hline GDP at market prices & 34,109 & 34,124 & 20,562 & 37,049 & 49,991 & 61,845 \\
\hline Oil GDP at market prices & 25,442 & 24,160 & 14,159 & 23,595 & 30,749 & 40,211 \\
\hline Non-oil GDP at market prices & 8,667 & 9,964 & 6,403 & 13,454 & 19,242 & 21,635 \\
\hline
\end{tabular}

Source: Iraqi authorities.

${ }^{*}$ Provisional estimates 
Table 2. Iraq: Gross Domestic Product at Constant 1998 Prices, 2000-06 (In billions of ID, unless otherwise indicated)

\begin{tabular}{|c|c|c|c|c|c|c|c|}
\hline & 2000 & 2001 & $2002^{*}$ & $2003^{*}$ & $2004^{*}$ & $2005^{*}$ & $2006^{*}$ \\
\hline Agriculture, forestry, hunting and fishing & 4,589 & 4,644 & 5,433 & 3,850 & 3,785 & 4,320 & 4,203 \\
\hline Mining and quarrying & 25,900 & 25,701 & 22,123 & 13,930 & 19,057 & 17,967 & 17,743 \\
\hline Crude oil & 25,878 & 25,676 & 22,099 & 13,917 & 19,035 & 17,942 & 17,711 \\
\hline Other types of mining & 23 & 25 & 24 & 13 & 22 & 25 & 31 \\
\hline Manufacturing industry & 1,748 & 1,909 & 1,740 & 1,244 & 895 & 956 & 1,009 \\
\hline Electricity and water & 379 & 435 & 504 & 200 & 424 & 451 & 516 \\
\hline Building and construction & 433 & 887 & 1,078 & 258 & 494 & 1,187 & 249 \\
\hline Transport, communications, and storage & 2,427 & 2,277 & 2,368 & 1,260 & 1,602 & 1,812 & 1,214 \\
\hline Wholesale, retail, hotels \& others & 1,770 & 2,309 & 1,862 & 1,056 & 1,334 & 1,295 & 2,003 \\
\hline Finance, insurance, real estate and business services & 1,116 & 1,154 & 1,189 & 1,120 & 1,201 & 1,259 & 1,294 \\
\hline Banking and insurance & 170 & 180 & 187 & 89 & 140 & 167 & 171 \\
\hline Ownership of dwelling & 946 & 974 & 1,002 & 1,031 & 1,061 & 1,092 & 1,124 \\
\hline Social and personal services & 4,144 & 4,176 & 4,209 & 4,242 & 4,501 & 5,149 & 8,226 \\
\hline Social services & 3,822 & 3,845 & 3,868 & 3,891 & 4,140 & 4,777 & 7,924 \\
\hline Personal services & 322 & 331 & 341 & 351 & 361 & 371 & 303 \\
\hline GDP at constant factor costs & 42,506 & 43,492 & 40,506 & 27,161 & 33,293 & 34,396 & 36,457 \\
\hline Less: Imputed banks service charge & 148 & 157 & 161 & 170 & 108 & 128 & 162 \\
\hline GDP at constant market prices & 42,359 & 43,335 & 40,345 & 26,990 & 33,186 & 34,268 & 36,296 \\
\hline
\end{tabular}

Source: Iraqi authorities.

* Provisional estimates. 
Table 3. Iraq: Crude Oil Production, 2004-06

(In thousands of barrels)

\begin{tabular}{|c|c|c|c|c|}
\hline & & 2004 & 2005 & 2006 \\
\hline \multirow[t]{13}{*}{ Total production } & January & 65,150 & 57,624 & 48,284 \\
\hline & February & 60,205 & 52,977 & 48,361 \\
\hline & March & 67,363 & 58,177 & 60,194 \\
\hline & April & 64,242 & 56,287 & 60,941 \\
\hline & May & 58,958 & 58,906 & 63,182 \\
\hline & June & 50,855 & 57,659 & 67,879 \\
\hline & July & 59,678 & 61,802 & 63,928 \\
\hline & August & 57,300 & 61,770 & 62,536 \\
\hline & September & 67,909 & 56,223 & 63,793 \\
\hline & October & 65,514 & 56,254 & 62,633 \\
\hline & November & 53,459 & 51,373 & 56,551 \\
\hline & December & 67,307 & 49,349 & 56,169 \\
\hline & Total & 737,940 & 678,401 & 714,451 \\
\hline \multirow[t]{13}{*}{ Southern oil } & January & 51,966 & 48,403 & 40,566 \\
\hline & February & 47,662 & 44,910 & 43,664 \\
\hline & March & 53,214 & 49,406 & 51,465 \\
\hline & April & 49,325 & 47,756 & 51,541 \\
\hline & May & 44,119 & 48,403 & 53,782 \\
\hline & June & 42,685 & 47,019 & 55,779 \\
\hline & July & 50,834 & 50,236 & 55,628 \\
\hline & August & 42,634 & 49,985 & 53,636 \\
\hline & September & 51,791 & 47,690 & 54,793 \\
\hline & October & 50,652 & 47,799 & 55,033 \\
\hline & November & 41,432 & 43,593 & 50,152 \\
\hline & December & 55,420 & 40,559 & 50,241 \\
\hline & Total & 581,734 & 565,759 & 616,280 \\
\hline \multirow[t]{13}{*}{ Northern oil company } & January & 13,184 & 9,221 & 7,718 \\
\hline & February & 12,543 & 8,067 & 4,697 \\
\hline & March & 14,149 & 8,771 & 8,729 \\
\hline & April & 14,917 & 8,531 & 9,400 \\
\hline & May & 14,839 & 10,503 & 9,400 \\
\hline & June & 8,170 & 10,640 & 12,100 \\
\hline & July & 8,844 & 11,566 & 8,300 \\
\hline & August & 14,666 & 11,785 & 8,900 \\
\hline & September & 16,118 & 8,533 & 9,000 \\
\hline & October & 14,862 & 8,455 & 7,600 \\
\hline & November & 12,027 & 7,780 & 6,399 \\
\hline & December & 11,887 & 8,790 & 5,928 \\
\hline & Total & 156,206 & 112,642 & 98,171 \\
\hline
\end{tabular}

Source: Iraqi authorities. 
Table 4. Iraq: Electricity-Average Monthly Production, 2001-06 (In megawatts)

\begin{tabular}{lcccccc}
\hline & 2001 & 2002 & 2003 & 2004 & 2005 & 2006 \\
\hline January & 3,366 & 3,932 & 4,463 & 3,306 & 3,439 & 3,620 \\
February & 3,477 & 3,793 & 4,431 & 3,545 & 3,720 & 3,654 \\
March & 2,921 & 3,231 & 3,852 & 3,384 & 3,488 & 3,913 \\
April & 2,861 & 3,280 & 711 & 3,048 & 3,411 & 3,687 \\
May & 3,127 & 3,804 & 2,136 & 3,135 & 3,676 & 3,743 \\
June & 3,720 & 4,388 & 2,818 & 3,621 & 4,262 & 4,407 \\
July & 4,005 & 4,601 & 2,929 & 3,995 & 4,429 & 4,477 \\
August & 4,177 & 4,766 & 3,018 & 4,304 & 4,108 & 4,465 \\
September & 3,888 & 4,273 & 3,107 & 4,119 & 4,189 & 4,279 \\
October & 3,283 & 4,004 & 3,265 & 4,130 & 3,801 & 4,108 \\
November & 3,220 & 3,759 & 2,938 & 3,247 & 3,681 & 3,922 \\
December & 3,488 & 4,132 & 2,976 & 3,320 & 3,737 & 3,987 \\
Total & 41,533 & 47,963 & 36,644 & 43,154 & 45,941 & 48,262 \\
& & & & & & \\
\hline
\end{tabular}

Source: Iraqi authorities. 
Table 5. Iraq: Cement Production, 2001-06

(In tons)

\begin{tabular}{rrrrr}
\hline & $\begin{array}{r}\text { White } \\
\text { Cement }\end{array}$ & $\begin{array}{r}\text { Normal } \\
\text { Cement }\end{array}$ & $\begin{array}{r}\text { Resistant } \\
\text { Cement }\end{array}$ & Total \\
\hline & & & & \\
2001 & 157.6 & $3,760.7$ & $1,549.4$ & $5,467.7$ \\
2002 & 174.9 & $4,897.5$ & $2,098.6$ & $7,171.0$ \\
2003 & 54.1 & $1,250.5$ & 523.4 & $1,828.0$ \\
2004 & 47.0 & $1,647.8$ & 486.8 & $2,181.6$ \\
2005 & 57.8 & $1,989.1$ & 514.4 & $2,561.3$ \\
2006 & 19.1 & $1,958.3$ & 536.2 & $2,513.6$ \\
& & & & \\
\hline
\end{tabular}

Source: Iraqi authorities. 

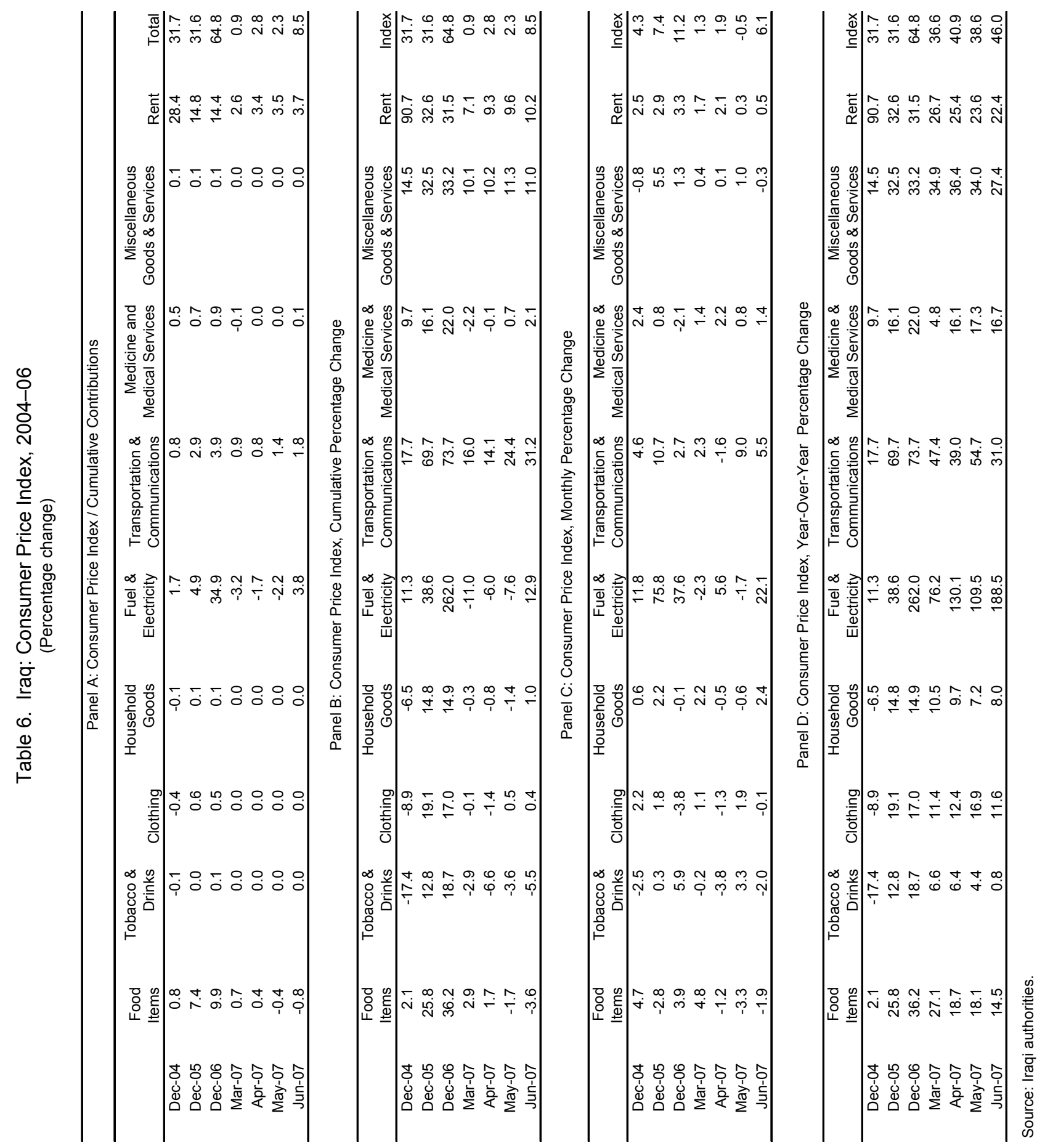
Table 7. Iraq: Exchange Rate, 2003-07

(In dinar per U.S. dollar)

\begin{tabular}{|c|c|c|c|c|}
\hline & & $\begin{array}{r}\text { Average exchange } \\
\text { rate in dinar per } \\
\text { US dollar }\end{array}$ & $\begin{array}{r}\text { Amount bought } \\
\text { by the Central Bank } \\
\text { of Iraq at auction } \\
\text { in millions of US dollars }\end{array}$ & $\begin{array}{l}\text { Amount sold } \\
\text { by the Central Bank } \\
\text { of Iraq at auction } \\
\text { in millions of US dollars }\end{array}$ \\
\hline \multirow[t]{3}{*}{2003} & October & 1,949 & 200.0 & 17.8 \\
\hline & November & 1,975 & 500.0 & 152.0 \\
\hline & December & 1,764 & 201.2 & 123.4 \\
\hline \multirow[t]{12}{*}{2004} & January & 1,487 & 513.9 & 16.0 \\
\hline & February & 1,413 & 402.7 & 157.3 \\
\hline & March & 1,419 & 651.9 & 401.8 \\
\hline & April & 1,440 & 814.1 & 366.7 \\
\hline & May & 1,461 & 551.6 & 366.8 \\
\hline & June & 1,458 & 600.4 & 239.1 \\
\hline & July & 1,460 & $1,600.0$ & 382.2 \\
\hline & August & 1,460 & 550.6 & 509.5 \\
\hline & September & 1,461 & 581.0 & 622.0 \\
\hline & October & 1,460 & 0.1 & $1,210.5$ \\
\hline & November & 1,460 & 4.3 & $1,189.5$ \\
\hline & December & 1,460 & 11.2 & 645.9 \\
\hline \multirow[t]{12}{*}{2005} & January & 1,460 & 4.0 & 391.1 \\
\hline & February & 1,460 & 24.9 & 286.7 \\
\hline & March & 1,461 & 2.9 & 648.5 \\
\hline & April & 1,465 & $1,100.2$ & 804.7 \\
\hline & May & 1,469 & $1,568.6$ & $1,800.7$ \\
\hline & June & 1,465 & 702.5 & 991.1 \\
\hline & July & 1,472 & 1.4 & 789.9 \\
\hline & August & 1,477 & 901.3 & $1,003.2$ \\
\hline & September & 1,476 & 2.6 & $1,055.0$ \\
\hline & October & 1,471 & 21.3 & 972.2 \\
\hline & November & 1,474 & 0.6 & 937.5 \\
\hline & December & 1,474 & 0.2 & 860.6 \\
\hline \multirow[t]{12}{*}{2006} & January & 1,479 & 1.3 & 887.2 \\
\hline & February & 1,477 & 401.5 & 950.8 \\
\hline & March & 1,477 & 4.2 & $1,173.9$ \\
\hline & April & 1,478 & 0.0 & 988.8 \\
\hline & May & 1,477 & 31.3 & 920.2 \\
\hline & June & 1,477 & & 861.3 \\
\hline & July & 1,477 & 0.5 & 876.0 \\
\hline & August & 1,477 & 12.9 & $1,047.5$ \\
\hline & September & 1,475 & 7.8 & $1,186.7$ \\
\hline & October & 1,471 & & $1,076.1$ \\
\hline & November & 1,453 & 13.1 & 898.4 \\
\hline & December & 1,391 & 37.8 & 307.6 \\
\hline \multirow[t]{6}{*}{2007} & January & 1,304 & 25.5 & $1,166.8$ \\
\hline & February & 1,285 & 20.9 & $1,223.0$ \\
\hline & March & 1,277 & 30.4 & $1,302.7$ \\
\hline & April & 1,271 & 40.9 & $1,376.6$ \\
\hline & May & 1,262 & 11.5 & $1,455.9$ \\
\hline & June & 1,256 & 16.7 & $1,235.3$ \\
\hline
\end{tabular}

Source: Iraqi authorities. 
Table 8. Iraq: Interest Rates, 2002-07

(In percent)

\begin{tabular}{|c|c|c|c|c|c|c|}
\hline & $\begin{array}{c}2002 \\
\text { Dec. }\end{array}$ & $\begin{array}{c}2003 \\
\text { Dec. }\end{array}$ & $\begin{array}{c}2004 \\
\text { Dec. }\end{array}$ & $\begin{array}{c}2005 \\
\text { Dec. }\end{array}$ & $\begin{array}{c}2006 \\
\text { Dec. }\end{array}$ & $\begin{array}{l}2007 \\
\text { June }\end{array}$ \\
\hline \multicolumn{7}{|l|}{ Ministry of Finance } \\
\hline Treasury bills (91-days) & 6.0 & 6.4 & 6.0 & 8.9 & 16.0 & 21.0 \\
\hline Overdraft & 20.0 & $\ldots$ & $\ldots$ & $\ldots$ & $\ldots$ & $\ldots$ \\
\hline \multicolumn{7}{|l|}{ Central Bank of Iraq (CBI) } \\
\hline Policy rate & $\ldots$ & $\ldots$ & 6.0 & 7.0 & 16.0 & 20.0 \\
\hline Primary credit rate & $\ldots$ & $\ldots$ & 8.0 & 9.0 & 18.0 & 22.0 \\
\hline Secondary credit rate & $\ldots$ & $\ldots$ & 9.0 & 10.0 & 19.0 & 23.0 \\
\hline Lender of last resort & $\ldots$ & $\ldots$ & 9.5 & 10.5 & 19.5 & 23.5 \\
\hline Overnight deposits & $\ldots$ & $\ldots$ & 4.0 & 5.0 & 14.0 & 18.0 \\
\hline ID 14-days deposits & $\ldots$ & $\ldots$ & $\ldots$ & 6.0 & 15.0 & 19.0 \\
\hline ID 30-days deposits & $\ldots$ & $\ldots$ & $\ldots$ & 7.0 & 16.0 & 20.0 \\
\hline USD overnight deposits & $\ldots$ & $\ldots$ & 2.0 & 2.5 & 2.8 & 2.8 \\
\hline USD 30 day deposits & $\ldots$ & $\ldots$ & $\ldots$ & 3.0 & 3.3 & 3.3 \\
\hline USD 90 day deposits & $\ldots$ & $\ldots$ & $\ldots$ & 3.3 & 3.5 & 3.5 \\
\hline \multicolumn{7}{|l|}{ CBI Bills } \\
\hline 182-days bills & $\ldots$ & $\ldots$ & $\ldots$ & $\ldots$ & 15.0 & 21.0 \\
\hline \multicolumn{7}{|l|}{ Commercial banks rates $1 /$} \\
\hline ID bank deposits (one year fixed) & 9.0 & 9.0 & 8.0 & 7.1 & 7.4 & 11.8 \\
\hline FX bank deposits (one year fixed) & $\ldots$ & $\ldots$ & 4.0 & 3.7 & 4.2 & 5.2 \\
\hline ID bank loans ( $1-5$ years $)$ & 15.0 & 15.0 & 13.0 & 14.0 & 15.7 & 19.4 \\
\hline FX bank loans ( $1-5$ years) & $\ldots$ & $\ldots$ & 8.6 & 10.9 & 12.6 & 15.0 \\
\hline
\end{tabular}

Source: Iraqi authorities.

1/ Commercial banks rates are as of end-May 2007 
Table 9. Iraq: Central Bank Survey, 2003-06

(In billions of Iraqi dinars, unless otherwise indicated)

\begin{tabular}{|c|c|c|c|c|}
\hline & $\begin{array}{l}2003 \\
\text { Dec. }\end{array}$ & $\begin{array}{c}2004 \\
\text { Dec. }\end{array}$ & $\begin{array}{l}2005 \\
\text { Dec. }\end{array}$ & $\begin{array}{c}2006 \\
\text { Dec. }\end{array}$ \\
\hline Net foreign exchange assets $1 /$ & 1,600 & 10,743 & 17,078 & 25,413 \\
\hline Foreign exchange assets & 1,916 & 11,538 & 17,724 & 26,043 \\
\hline Gold 2/ & 114 & 115 & 143 & 159 \\
\hline Other & 1,803 & 11,422 & 17,581 & 25,885 \\
\hline $\mathrm{CBI}$ vaults & 1,803 & 10,361 & 16,600 & 24,962 \\
\hline SDR Account 3/ & 0 & 1,061 & 982 & 922 \\
\hline Foreign exchange liabilities & -316 & -795 & -646 & -630 \\
\hline Of which: Borrowing from the MF & $\ldots$ & -637 & -637 & -637 \\
\hline Net domestic assets & 4,038 & 1,485 & $-3,380$ & $-7,749$ \\
\hline Domestic assets & 3,698 & 4,345 & 138 & -308 \\
\hline Net claims on general government & 3,698 & 4,336 & 128 & -320 \\
\hline Holdings of treasury bills & 2,874 & 2,589 & 2,560 & 4,675 \\
\hline Holdings of discounted treasury bills & 206 & 709 & 707 & 719 \\
\hline Overdrafts & 909 & 1,637 & 1,821 & 0 \\
\hline Domestic currency deposits & -15 & -596 & $-3,577$ & $-5,170$ \\
\hline Foreign currency deposits & -276 & -3 & $-1,382$ & -544 \\
\hline Claims on commercial banks & 0 & 9 & 9 & 12 \\
\hline Monetary policy instruments 4/ & 0 & 0 & $-3,731$ & $-8,075$ \\
\hline Other items net & 339 & $-2,859$ & 214 & 635 \\
\hline Reserve money & 5,638 & 12,228 & 13,698 & 17,664 \\
\hline Currency outside banks & 2,606 & 7,163 & 9,110 & 10,789 \\
\hline Banks reserves & 1,052 & 4,208 & 3,442 & 5,748 \\
\hline Required & 717 & 2,287 & 2,596 & 3,572 \\
\hline Excess & 335 & 1,921 & 845 & 2,176 \\
\hline \multicolumn{5}{|l|}{ Memorandum items: } \\
\hline Reserve money (annual growth, in percent) & $\ldots$ & 116.9 & 12.0 & 29.0 \\
\hline Currency issued (annual growth, in percent) & $\ldots$ & 74.9 & 27.9 & 16.2 \\
\hline Gross foreign exchange assets (in millions of U.S. dollars) & 1,134 & 7,902 & 12,024 & 18,723 \\
\hline Foreign exchange liabilities (in millions of U.S. dollars) & -187 & -547 & -440 & -453 \\
\hline Net foreign exchange assets (in millions of U.S. dollars) & 947 & 7,355 & 11,584 & 18,270 \\
\hline Exchange rate (period average) & 1,690 & 1,453 & 1,469 & 1,467 \\
\hline Gross foreign exchange assets/reserve money (in percent) & 34.0 & 94.4 & 129.4 & 147.4 \\
\hline Claims on banks/reserve money (in percent) & 0.0 & 0.1 & 0.1 & 0.1 \\
\hline Reserve money coverage (in percent) & 28.4 & 87.8 & 124.7 & 143.9 \\
\hline Currency outside banks/Reserve money (in percent) & 46.2 & 58.6 & 66.5 & 61.1 \\
\hline Currency outside banks/Non-oil GDP (in percent) & 32.6 & 59.3 & 52.3 & 35.0 \\
\hline Ratio of Currency outside banks to GDP & 10.6 & 19.1 & 18.4 & 14.6 \\
\hline Ratio of Reserve money to GDP & 15.2 & 32.7 & 27.7 & 23.9 \\
\hline
\end{tabular}

Sources: Iraqi authorities; and staff estimates.

1/ Valued at market exchange rates.

2/ For 2005, valued at market prices.

3/ This includes both SDR holdings and the reserve tranche position.

4/ This mainly represents the ID and US\$ overnight standing deposit facilities. 
Table 10. Iraq: Depository Corporations Survey, 2004-06 (In billions of Iraqi dinars, unless otherwise indicated)

\begin{tabular}{lrrr}
\hline & 2004 & 2005 & 2006 \\
\hline Net foreign assets & Dec. & Dec. & Dec. \\
$\quad$ Claims on nonresidents & $-5,944$ & 15 & 10,889 \\
$\quad$ less: Liabilities to nonresidents & 18,440 & 27,470 & 37,264 \\
Domestic claims & $-24,384$ & $-27,456$ & $-26,375$ \\
$\quad$ Net claims on central government & 2,711 & $-1,204$ & $-4,458$ \\
$\quad$ Claims on central government & 1,917 & $-2,936$ & $-7,314$ \\
$\quad$ less: Liabilities to central government & 6,379 & 6,719 & 7,535 \\
Claims on other sectors & $-4,462$ & $-9,655$ & $-14,850$ \\
Other items net & 794 & 1,732 & 2,856 \\
& 17,589 & 16,077 & 13,041 \\
Broad money liabilities & & & \\
$\quad$ Currency outside depository corporations & 14,356 & 14,888 & 19,472 \\
$\quad$ Transferable deposits & 6,734 & 9,113 & 10,968 \\
Other deposits & 6,287 & 3,871 & 6,247 \\
& 1,334 & 1,904 & 2,257 \\
Broad money liabilities & $($ Percent change) & \\
Currency outside depository corporations & $\ldots$ & 3.7 & 30.8 \\
Claims on other sectors & $\ldots$ & 35.3 & 20.4 \\
& $\ldots$ & 118.1 & 64.9 \\
\hline
\end{tabular}

Sources: Iraqi authorities, and Fund staff estimates and projections. 
Table 11. Iraq: Fiscal and Oil Sector Accounts, 2004-06

(Economic classification; in billions of ID)

\begin{tabular}{|c|c|c|c|}
\hline & 2004 & 2005 & $\begin{array}{r}2006 \\
\text { Prel. }\end{array}$ \\
\hline Revenues and Grants & 29,784 & 49,505 & 58,497 \\
\hline Revenues & 26,376 & 36,655 & 49,430 \\
\hline Crude oil export revenues & 25,108 & 33,896 & 43,736 \\
\hline Revenues of oil-related state owned enterprises & 799 & 1,456 & 3,611 \\
\hline Tax revenues & 160 & 495 & 585 \\
\hline Direct taxes & 78 & 207 & 345 \\
\hline Indirect taxes & 81 & 288 & 240 \\
\hline Non-tax revenues & 309 & 809 & 1,498 \\
\hline Grants & 3,408 & 12,850 & 9,067 \\
\hline Expenditures & 45,202 & 44,497 & 49,756 \\
\hline Current expenditures & 36,887 & 35,560 & 40,188 \\
\hline Salary and pension & 4,532 & 9,282 & 10,959 \\
\hline Goods and Services (non-oil sector) & 10,998 & 13,019 & 12,193 \\
\hline Budgeted goods and services & 6,985 & 3,135 & 5,818 \\
\hline Financed by oil-for-food program (OFFP) (other than social safety net) & 1,616 & 760 & 220 \\
\hline Overhead costs for donors' financed projects $1 /$ & 860 & 3,512 & 2,588 \\
\hline Other goods and services financed by donors $2 /$ & 1,538 & 5,612 & 3,567 \\
\hline Goods and Services (oil sector) & 5,609 & 5,212 & 6,560 \\
\hline Oil derivative imports & 4,823 & 4,128 & 4,082 \\
\hline Operating expenditures of oil-related state-owned enterprises & 786 & 1,085 & 2,477 \\
\hline Transfers & 14,075 & 6,232 & 7,928 \\
\hline Social safety net (excluding OFFP) & 3,829 & 3,751 & 5,500 \\
\hline Social safety net (financed by OFFP) & 2,116 & 0 & 0 \\
\hline Transfers to SOEs & 1,237 & 900 & 1,299 \\
\hline Other transfers & 6,893 & 1,581 & 1,129 \\
\hline Interest payments & 369 & 86 & 313 \\
\hline Domestic interest payments & 369 & 86 & 182 \\
\hline External interest payments & 0 & 0 & 131 \\
\hline War reparations & 1,304 & 1,730 & 2,235 \\
\hline Investment expenditures & 8,314 & 8,936 & 9,464 \\
\hline Non-oil investment expenditures & 6,126 & 6,587 & 7,703 \\
\hline Domestic financed reconstruction expenditure & 4,087 & 2,189 & 4,693 \\
\hline OFFP financed reconstruction expenditure & 1,079 & 478 & 0 \\
\hline Grant financed reconstruction expenditure & 961 & 3,448 & 2,563 \\
\hline Oil investment expenditures (including on refineries) & 2,188 & 2,349 & 1,761 \\
\hline Contingency & 0 & 0 & 104 \\
\hline Balance (including grants) & $-15,417$ & 5,008 & 8,741 \\
\hline Balance (excluding grants) & $-18,826$ & $-7,842$ & -326 \\
\hline Change in outstanding Letters of Credit [+ increase] $3 /$ & $\ldots$ & 2,947 & 1,672 \\
\hline Financing, of which & & $-2,095$ & $-6,354$ \\
\hline External financing & 15,426 & 2,721 & $-3,522$ \\
\hline Assets held abroad & 10,127 & 1,462 & $-2,147$ \\
\hline Other financing $4 /$ & 5,969 & 1,486 & 458 \\
\hline Amortization & 670 & 227 & 1,833 \\
\hline Domestic financing & -533 & $-4,816$ & $-2,833$ \\
\hline Bank financing & -333 & $-5,124$ & $-1,884$ \\
\hline Non bank financing & -200 & 308 & -949 \\
\hline Residual or Financing gap $[+] /$ surplus $[-]$ & 525 & 34 & -161 \\
\hline
\end{tabular}

Sources: Iraqi authorities; and Fund staff estimates.

1/ Overhead costs associated with donor-financed reconstruction.

2/ Other goods and services financed by donors include security spending associated with the implementation of reconstruction projects.

3/ LCs in the Trade Bank of Iraq, for which 100 percent down-payment is customarily required.

4 / Includes financing from LCs previously issued under the UN oil-for-food program. 
Table 12. Iraq: Government Current Expenditure by Source, 2004-06

(Administrative classification; in billions of ID)

\begin{tabular}{|c|c|c|c|}
\hline & 2004 & 2005 & 2006 \\
\hline Financial Surveillance Office & 7 & 16 & 19 \\
\hline Ministry of Foreign Affairs & 10 & 77 & 93 \\
\hline Ministry of Finance & 22,685 & 15,806 & 18,490 \\
\hline Ministry of Internal Affairs & 630 & 1,415 & 2,318 \\
\hline Ministry of Labor and Social Affairs & 35 & 152 & 74 \\
\hline Ministry of Health & 577 & 720 & 827 \\
\hline Ministry of Security and National Defense & 245 & 1,649 & 1,814 \\
\hline Ministry of Justice & 67 & 71 & 83 \\
\hline Ministry of Education & 1,059 & 1,407 & 1,546 \\
\hline Ministry of Youth and Sports & 11 & 31 & 34 \\
\hline Ministry of Trade & 6 & 27 & 32 \\
\hline Ministry of Culture & 10 & 27 & 35 \\
\hline Ministry of Transportation & 12 & 71 & 93 \\
\hline Ministry of Public Works and Municipalities & 74 & 160 & 182 \\
\hline Ministry of Housing and Construction & 50 & 142 & 196 \\
\hline Ministry of Agriculture & 64 & 206 & 66 \\
\hline Ministry of Financial Resources & 68 & 240 & 226 \\
\hline Ministry of Petroleum & 354 & 240 & 271 \\
\hline Ministry of Planning and Development Cooperation & 14 & 12 & 53 \\
\hline Ministry of Industry and Mining & 8 & 19 & 29 \\
\hline Minintry of Higher Education and Academic Research & 258 & 390 & 528 \\
\hline Ministry of Religious Endowments \& Religious Affairs & 22 & 76 & 86 \\
\hline Baghdad Secretariat & 8 & $\ldots$ & $\ldots$ \\
\hline Ministry of Electricity & 49 & 220 & 429 \\
\hline Ministry of Science and Technology & 32 & 52 & 52 \\
\hline Ministry of Communications & 1 & 9 & 11 \\
\hline Ministry of the Environment & 5 & 8 & 13 \\
\hline Ministry of Immigration and Emigration & 3 & 4 & 4 \\
\hline Ministry of Human Rights & 2 & 4 & 4 \\
\hline Council of Justice & 7 & $\ldots$ & $\ldots$ \\
\hline Council of Judges (General Secretariat) & 10 & 51 & 71 \\
\hline National Aseembly & $\ldots$ & 70 & 81 \\
\hline Presidency & $\ldots$ & 69 & 98 \\
\hline Council of Ministers & $\ldots$ & 62 & 96 \\
\hline Kurdistan Regional Government & $\ldots$ & 3,910 & 4,508 \\
\hline Unclassified 3/ & 3,591 & $\ldots$ & $\ldots$ \\
\hline Total & 29,972 & 27,413 & 32,462 \\
\hline
\end{tabular}

Source: Iraqi authorities. 
Table 13. Iraq: Government Capital Expenditure by Source, 2004-06

(Administrative classification; in billions of ID)

\begin{tabular}{|c|c|c|c|}
\hline & 2004 & 2005 & 2006 \\
\hline Financial Surveillance Office & 4 & 2 & 2 \\
\hline Ministry of Foreign Affairs & 6 & 3 & 1 \\
\hline Ministry of Finance & 715 & 67 & 161 \\
\hline Ministry of Internal Affairs & 203 & $\ldots$ & 15 \\
\hline Ministry of Labor and Social Affairs & 8 & 10 & 8 \\
\hline Ministry of Health & 71 & 27 & 26 \\
\hline Ministry of Security and National Defense & 647 & $\ldots$ & 1 \\
\hline Ministry of Justice & 14 & 1 & 1 \\
\hline Ministry of Education & 10 & 2 & 24 \\
\hline Ministry of Youth and Sports & 7 & 13 & 10 \\
\hline Ministry of Trade & 3 & 10 & 7 \\
\hline Ministry of Culture & 11 & 5 & 1 \\
\hline Ministry of Transportation & 47 & 22 & 5 \\
\hline Ministry of Public Works and Municipalities & 121 & 101 & 122 \\
\hline Ministry of Housing and Construction & 85 & 77 & 136 \\
\hline Ministry of Agriculture & 14 & 1 & 3 \\
\hline Ministry of Financial Resources & 85 & 180 & 149 \\
\hline Ministry of Petroleum & 980 & 165 & 190 \\
\hline Ministry of Planning and Development Cooperation & 37 & 1 & 35 \\
\hline Ministry of Industry and Mining & 1 & 1 & 7 \\
\hline Minintry of Higher Education and Academic Research & 26 & 11 & 25 \\
\hline Ministry of Religious Endowments \& Religious Affairs & 8 & 10 & 13 \\
\hline Baghdad Secretariat & 0 & $\ldots$ & $\ldots$ \\
\hline Ministry of Electricity & 1,351 & 214 & 400 \\
\hline Ministry of Science and Technology & 8 & 8 & 5 \\
\hline Ministry of Communications & 21 & 0 & 1 \\
\hline Ministry of the Environment & 1 & 1 & 1 \\
\hline Ministry of Immigration and Emigration & 2 & 0 & 0 \\
\hline Ministry of Human Rights & 1 & 0 & 0 \\
\hline Council of Justice & 0 & 0 & 0 \\
\hline Council of Judges (General Secretariat) & 2 & 0 & 4 \\
\hline Kurdistan Regional Government & $\ldots$ & 772 & 790 \\
\hline Unclassified & 577 & $\ldots$ & $\ldots$ \\
\hline Total & 5,066 & 1,704 & 2,143 \\
\hline
\end{tabular}

Source: Iraqi authorities. 
Table 14. Iraq: Balance of Payments, 2004-06 1/ (In millions of U.S. dollars, unless otherwise indicated)

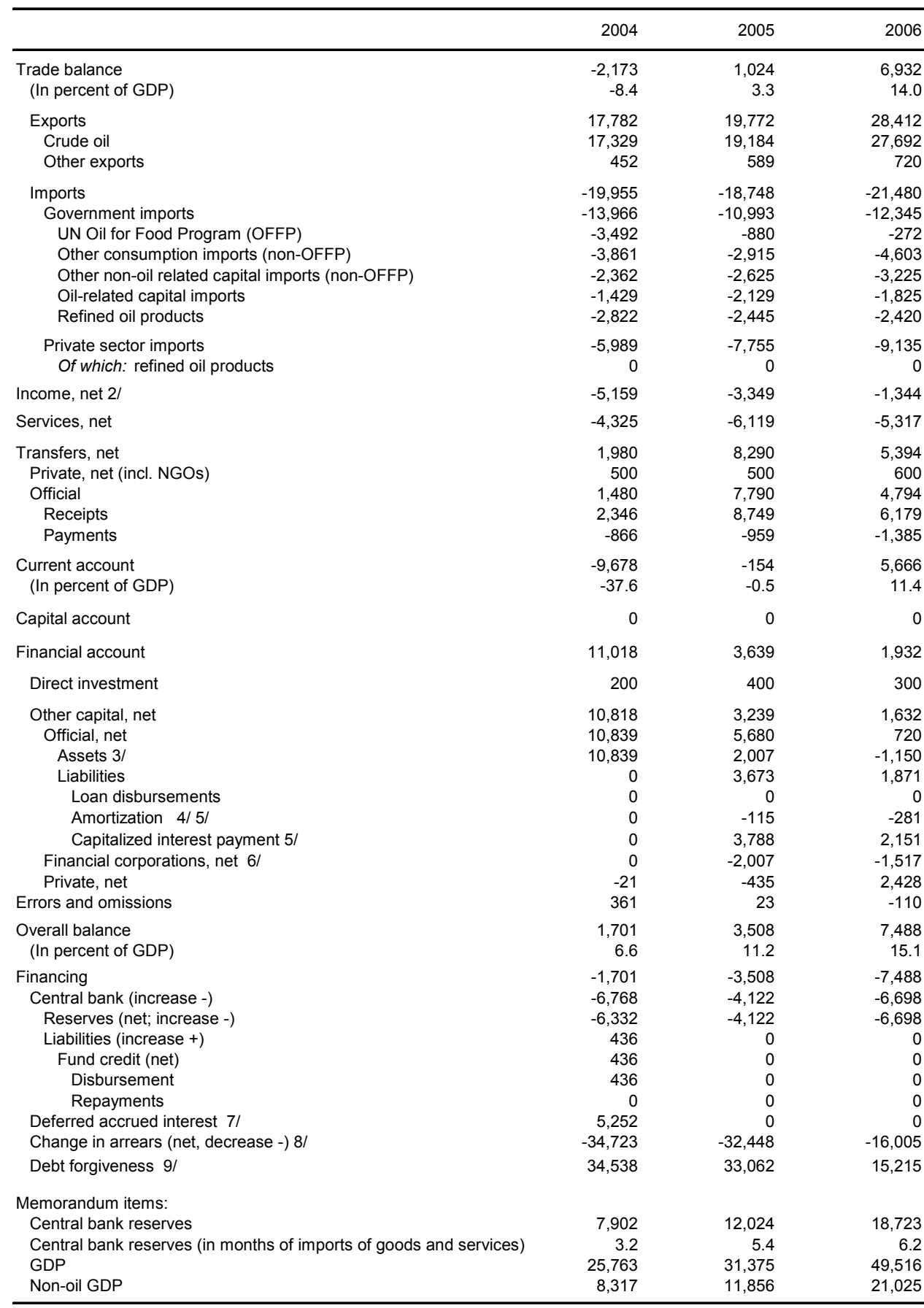

Sources: Iraqi authorities; and Fund staff estimates.

1/ Excludes U.S. military spending in Iraq.

2/ Includes interest accrued, deferred, and capitalized.

$3 /$ Includes the use/accumulation of the DFI ressources and the UN Oil for Food letters of credit.

4/ Includes the estimates of cash payments to settle the debt owed to small private creditors through cash exchange offers 5/ Based on the 2004 Paris Club agreement, the payments of principal and most interest during 2005-10 are deferred and capitalized.

6/ Reflects advance transfers for the execution of letter of credit (L/C).

7/ Estimates of accrued interest on existing stock of debt prior to Paris Club agreement.

$8 /$ Includes debt forgiveness and clearance of arrears on multilateral debt and arrears related to fuel imports from Turkey.

9/ The notional debt forgiveness reflects: (i) the first stage of debt reduction (30 percent) at end 2004; (ii) the second stage reduction (30 percent) at end-2005; and (iii) the settlement of debt owed to private creditors through cash and debt exchanges. Assumes debt reduction comparable to the Paris Club agreement on other debt-to-official creditors. 
Table 15. Iraq: Oil Exports, 2003-07

\begin{tabular}{|c|c|c|c|c|c|}
\hline & & $\begin{array}{r}\text { In millions of } \\
\text { U.S. dollars }\end{array}$ & $\begin{array}{r}\text { In } \\
\text { barrels }\end{array}$ & $\begin{array}{l}\text { In millions of } \\
\text { barrels per day }\end{array}$ & $\begin{array}{l}\text { In U.S. dollars } \\
\text { per barrel }\end{array}$ \\
\hline \multirow[t]{3}{*}{2003} & Third quarter & 1,066 & $\ldots$ & $\ldots$ & $\ldots$ \\
\hline & Fourth quarter & 2,855 & $\ldots$ & $\ldots$ & $\ldots$ \\
\hline & Total & 3,921 & $\ldots$ & $\ldots$ & $\ldots$ \\
\hline \multirow[t]{5}{*}{2004} & First quarter & 3,697 & $134,529,355$ & 1.48 & 26.78 \\
\hline & Second quarter & 4,440 & $143,357,973$ & 1.58 & 30.23 \\
\hline & Third quarter & 4,270 & $124,146,035$ & 1.35 & 33.37 \\
\hline & Fourth quarter & 4,923 & $138,820,244$ & 1.51 & 36.02 \\
\hline & Total & 17,330 & $540,853,607$ & 1.48 & 31.60 \\
\hline \multirow[t]{5}{*}{2005} & First quarter & 4,363 & $134,120,210$ & 1.49 & 32.61 \\
\hline & Second quarter & 5,332 & $122,467,980$ & 1.35 & 41.98 \\
\hline & Third quarter & 7,096 & $134,400,310$ & 1.46 & 51.83 \\
\hline & Fourth quarter & 6,331 & $127,218,424$ & 1.38 & 49.26 \\
\hline & Total & 23,122 & $518,206,924$ & 1.42 & 43.92 \\
\hline \multirow[t]{12}{*}{2006} & January & 1,549 & $31,659,562$ & 1.02 & 47.94 \\
\hline & February & 1,624 & $31,092,736$ & 1.11 & 50.54 \\
\hline & March & 2,263 & $42,618,728$ & 1.37 & 50.86 \\
\hline & April & 2,211 & $40,847,293$ & 1.36 & 54.43 \\
\hline & May & 2,901 & $47,795,797$ & 1.54 & 59.85 \\
\hline & June & 2,682 & $43,979,043$ & 1.47 & 59.91 \\
\hline & July & 3,226 & $51,950,027$ & 1.68 & 62.13 \\
\hline & August & 3,315 & $52,990,408$ & 1.71 & 64.08 \\
\hline & September & 2,842 & $48,051,719$ & 1.60 & 59.37 \\
\hline & October & 2,637 & $50,253,335$ & 1.62 & 52.07 \\
\hline & November & 2,073 & $39,079,127$ & 1.30 & 49.12 \\
\hline & December & 2,477 & $47,753,855$ & 1.54 & 52.14 \\
\hline \multirow[t]{5}{*}{2006} & First quarter & 5,436 & $105,371,026$ & 1.17 & 49.78 \\
\hline & Second quarter & 7,795 & $132,622,133$ & 1.46 & 58.06 \\
\hline & Third quarter & 9,383 & $152,992,154$ & 1.66 & 61.86 \\
\hline & Fourth quarter & 7,188 & $137,086,317$ & 1.49 & 51.11 \\
\hline & Total & 29,802 & $528,071,630$ & 1.44 & 55.20 \\
\hline \multirow[t]{3}{*}{2007} & January & 2,246 & $45,252,005$ & 1.46 & 50.15 \\
\hline & February & 1,659 & $34,062,003$ & 1.22 & 48.87 \\
\hline & March & 2,451 & $48,470,458$ & 1.56 & 50.56 \\
\hline 2007 & First quarter & 6,356 & $127,784,466$ & 1.41 & 49.86 \\
\hline
\end{tabular}

Source: Iraqi authorities. 
Table 16. Iraq: Non-Oil Exports, 2004-06

(F.O.B., in thousand of U.S. dollars)

\begin{tabular}{|c|c|c|c|}
\hline & 2004 & 2005 & $\begin{array}{c}2006 \\
\text { Prel. }\end{array}$ \\
\hline Dates & 55,599 & 58,000 & 69,320 \\
\hline Books & 7,288 & 0 & 0 \\
\hline Habaya & 4,797 & 0 & 23 \\
\hline Clover & 4,677 & 261 & 691 \\
\hline Skins & 4,581 & 9,907 & 7,830 \\
\hline Pig Iron & 3,667 & 0 & 0 \\
\hline Mash bean & 3,432 & 1,339 & 2,533 \\
\hline Wool & 3,228 & 4,669 & 5,527 \\
\hline Cotton & 2,441 & 0 & 0 \\
\hline Licorice roots & 2,048 & 4,965 & 2,994 \\
\hline Painting solvent & 1,661 & 2,453 & 1,152 \\
\hline Bulgur wheat & 1,078 & 130 & 0 \\
\hline Rice & 983 & 59 & 0 \\
\hline Sesame & 913 & 0 & 0 \\
\hline Dried clover & 909 & 0 & 0 \\
\hline Thinner & 901 & 403 & 0 \\
\hline Cheese & 788 & 0 & 0 \\
\hline Wood & 767 & 0 & 0 \\
\hline Cement & 561 & 0 & 0 \\
\hline Date molasses & 553 & 438 & 253 \\
\hline Fertilizer & 521 & 320 & 92 \\
\hline Aniseed & 417 & 0 & 230 \\
\hline Paints & 388 & 0 & 0 \\
\hline Truffle & 384 & 0 & 0 \\
\hline Corn & 297 & 0 & 0 \\
\hline Cotton seeds & 224 & 0 & 0 \\
\hline Ink solvent & 222 & 0 & 0 \\
\hline Canning machines & 191 & 0 & 0 \\
\hline Broad bean & 168 & 0 & 69 \\
\hline Silvanos Acid & 166 & 498 & 0 \\
\hline Seeds & 151 & 664 & 0 \\
\hline Sulfur & 129 & 7,311 & 0 \\
\hline Other commodities $1 /$ & 1,198 & 27,154 & 139,585 \\
\hline Total & 105,329 & 118,571 & 230,299 \\
\hline
\end{tabular}

Source: Iraqi authorities.

1/ Includes unclassified items. 
Table 17. Iraq: Exports by Countries of Destination and SITC Classification, 2003-06 (F.O.B., in millions of U.S. dollars) $1 /$

\begin{tabular}{|c|c|c|c|c|c|}
\hline & & 2003 & 2004 & 2005 & $\begin{array}{c}2006 \\
\text { Prel. }\end{array}$ \\
\hline \multicolumn{2}{|c|}{ Total exports } & 10,082 & 17,782 & 23,697 & 30,528 \\
\hline \multicolumn{6}{|c|}{ By countries of destination } \\
\hline \multicolumn{2}{|c|}{ Arab countries } & 887 & 1,557 & 763 & 946 \\
\hline \multicolumn{2}{|c|}{ North and South American countries } & 7,179 & 10,792 & 13,185 & 17,035 \\
\hline \multicolumn{2}{|c|}{ European Union countries } & 1,341 & 3,127 & 6,277 & 6,808 \\
\hline \multicolumn{2}{|c|}{ Other European countries } & 101 & 132 & 393 & 611 \\
\hline \multicolumn{2}{|c|}{ Asian countries } & 282 & 2,155 & 2,292 & 5,098 \\
\hline \multicolumn{2}{|c|}{ Other countries } & 292 & 20 & 787 & 30 \\
\hline \multicolumn{6}{|c|}{ By SITC classification } \\
\hline \multicolumn{2}{|c|}{ SITC Description } & & & & \\
\hline 0 & Food and live animals & 504 & 36 & 60 & 86 \\
\hline 1 & Beverages and tobacco & 0 & 0 & 0 & 0 \\
\hline 2 & Crude materials, inedible, except fuels & 807 & 53 & 44 & 46 \\
\hline 3 & Mineral fuels, lubricants, and related materials & 8,459 & 17,675 & 23,578 & 30,298 \\
\hline 4 & Animal and vegetable oils, fats, and waxes & 50 & 0 & 0 & 0 \\
\hline 5 & Chemicals and related products & 101 & 0 & 0 & 2 \\
\hline 6 & Manufactured goods classified chiefly by materials & 91 & 18 & 15 & 16 \\
\hline 7 & Machinery and transport equipment & 10 & 0 & 0 & 72 \\
\hline 8 & Miscellaneous manufactured articles & 60 & 0 & 0 & 0 \\
\hline 9 & Commodities not classified anywhere in the SITC & 0 & 0 & 0 & 8 \\
\hline
\end{tabular}

Source: Iraqi authorities.

1/ The data may differ from those in the Balance of Payment due to different data collection, definition, and classification. 


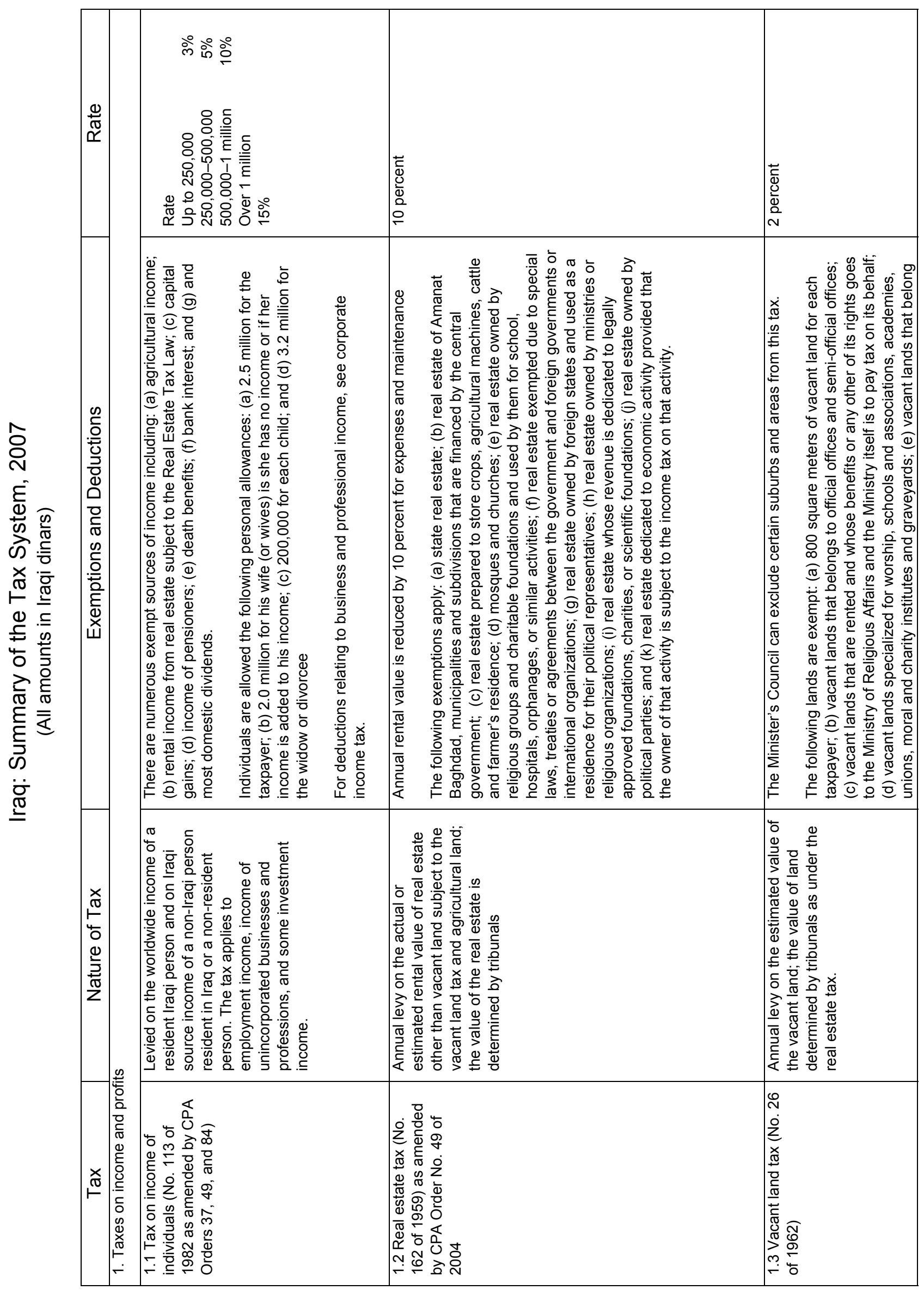




\begin{tabular}{|c|c|c|c|c|c|c|c|c|c|c|}
\hline$\frac{\Phi}{\frac{\Phi}{\widetilde{\varpi}}}$ & & 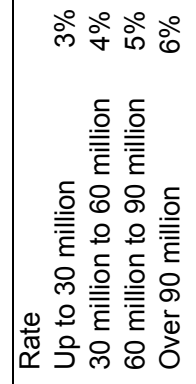 & 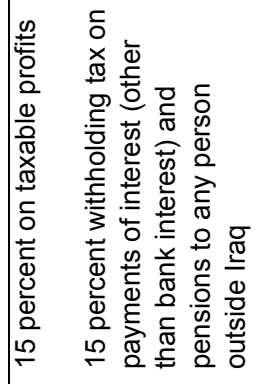 & & & & & & & \\
\hline 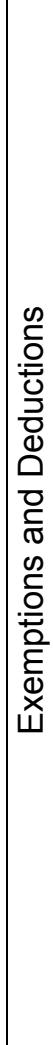 & 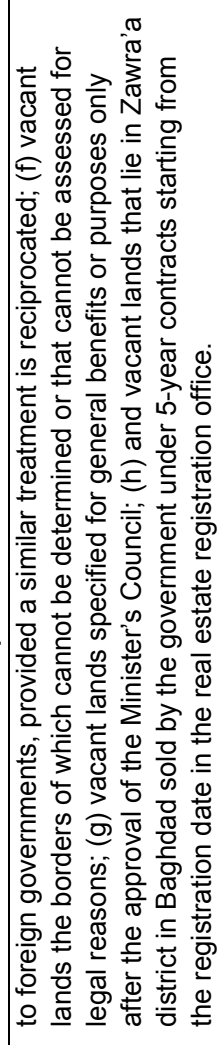 & 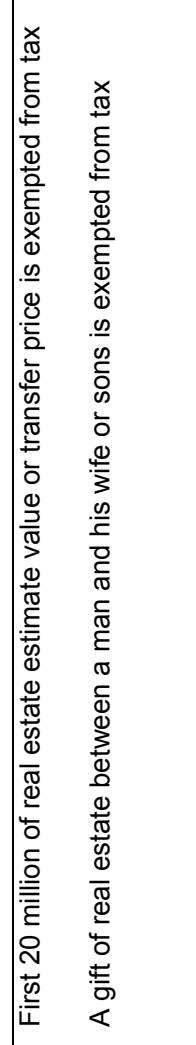 & 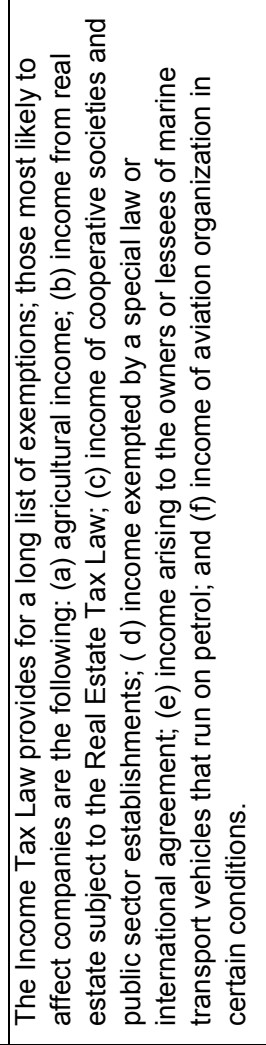 & 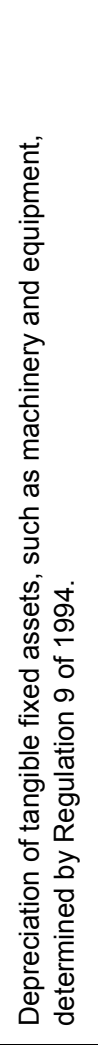 & 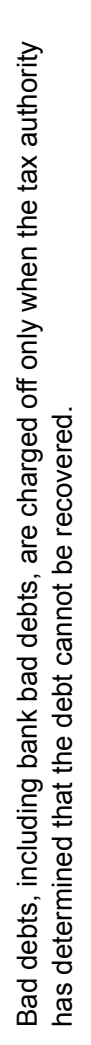 & 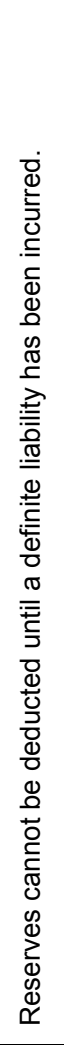 & 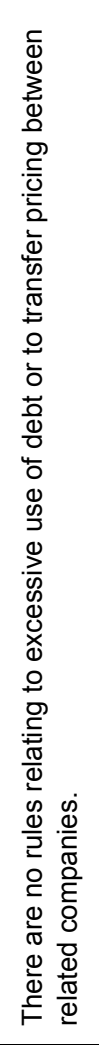 & 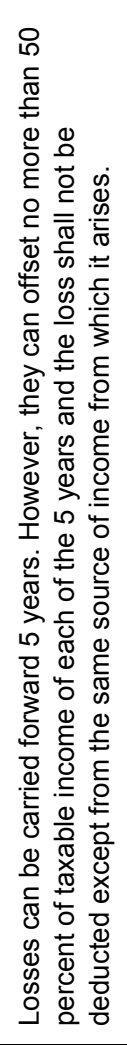 & 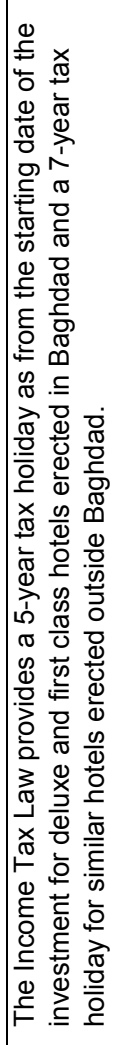 & 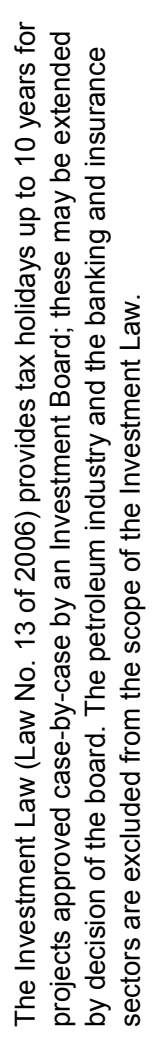 \\
\hline 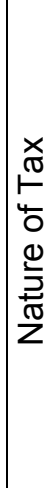 & & 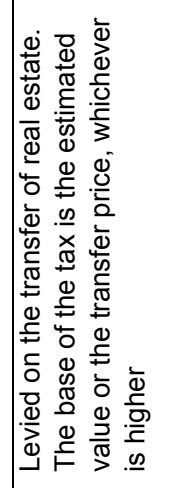 & 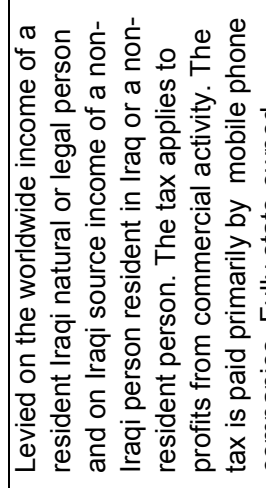 & 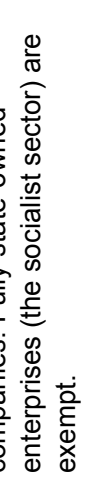 & & & & & & \\
\hline $\begin{array}{l}\underset{\sigma}{\sigma} \\
r\end{array}$ & & 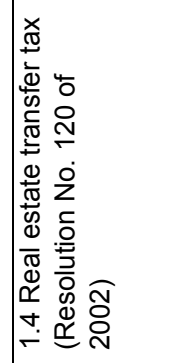 & 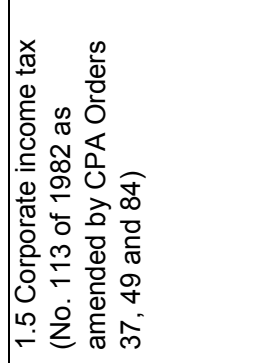 & & & & & & & \\
\hline
\end{tabular}




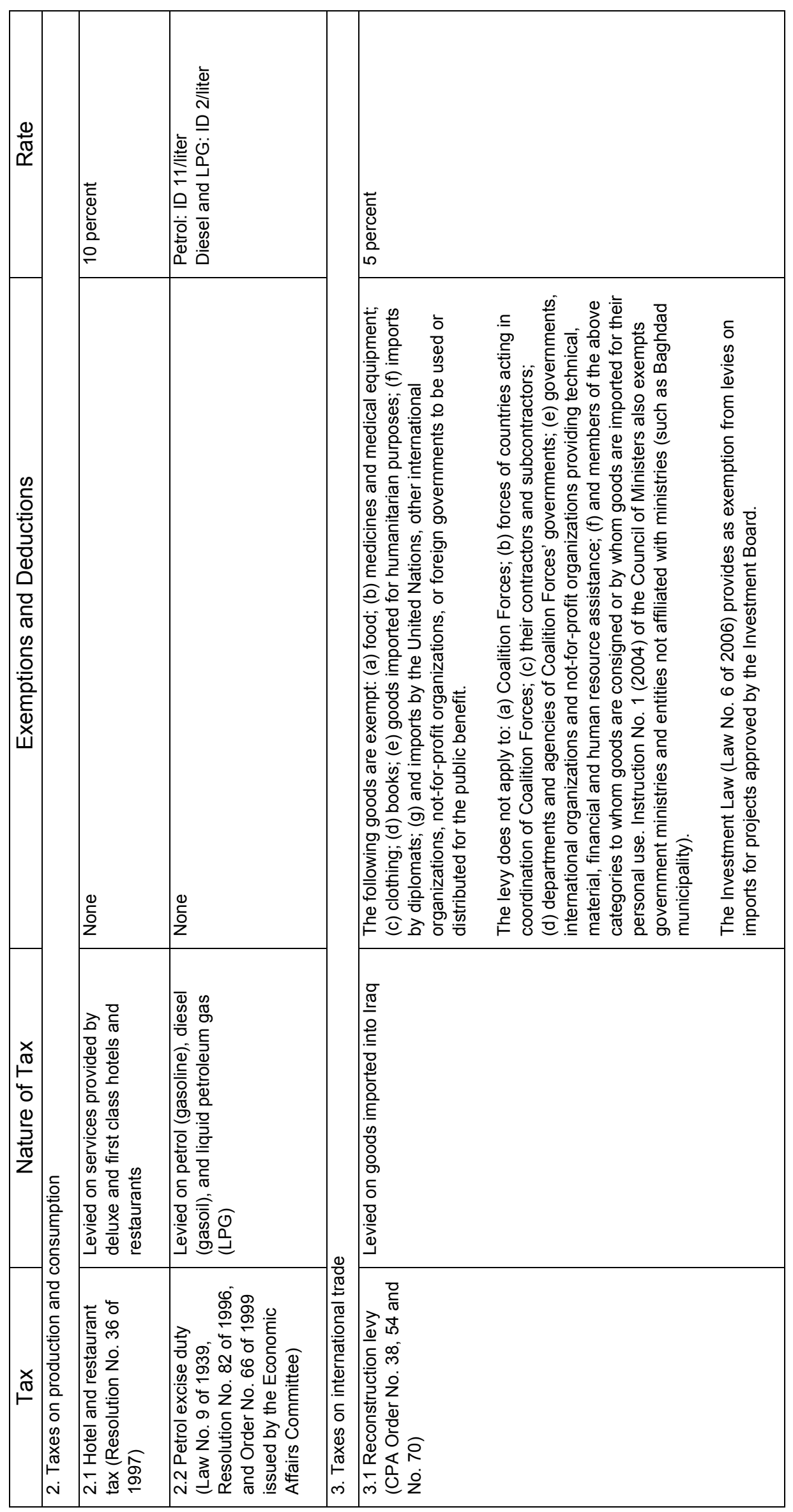

